\title{
PSI-SERIES, SINGULARITIES OF SOLUTIONS AND INTEGRABILITY OF POLYNOMIAL SYSTEMS
}

\author{
ARNAU MIR AND AMADEU DELSHAMS
}

\begin{abstract}
Psi-series (i.e., logarithmic series) of $m$-dimensional polynomial systems are considered. Its existence and convergence is studied, and an algorithm of location of logarithmic singularities is developed. Moreover, the relationship between psi-series and nonintegrability is stressed and in particular it is stated that $m$-dimensional polynomial systems with psi-series which do not reduce to Laurent series do not have $m-1$ independent algebraic first integrals.
\end{abstract}

\section{Introduction}

The integrability of a system of differential equations seems to be very well related with the kind of expansions of its solutions near singularities. Painlevé [2] gave a test to obtain integrable systems, based on looking for systems such that these expansions were simply Laurent series. There are several partial results supporting this test [1], as well as counterexamples [5].

A first generalization of the Laurent series is given by the so called psi-series:

$$
x(t)=\sum_{n \geq-k} p_{n}\left(\log \left(\frac{1}{\tau}\right)\right) \tau^{n},
$$

where $p_{n}$ are vector polynomials and $\tau=t-t_{p}$, near a singularity $t_{p}$ of a solution $x(t)$. To differentiate them from the standard Laurent series, we will call genuine psi-series those psi-series such that some $p_{n}$ is not a constant.

In many cases, these expansions appear in non-integrable systems. However, there are cases of integrable systems with genuine psi-series. In a previous work [4], we have studied all quadratic systems with a center at the origin. It turns out that in all the cases that genuine psi-series appear, the general integral is not an algebraic function. We will see here that this is the general situation, i.e., that the existence of genuine psi-series is an obstruction for algebraic integrals.

In section 2, we give conditions for the existence of psi-series for $m$-dimensional polynomial systems. Section 3 is devoted to the convergence of psi-series and an estimate of the region of convergence is given. The psi-series expansion is an important tool to locate singularities. This tool is explained in section 4. 
From the study developed there, one gathers that

- the Painlevé test cannot detect polynomial integrable systems if some of the first integrals are transcendent functions,

- any $m$-dimensional polynomial system with a complete solution in terms of genuine psi-series does not have $m-1$ independent algebraic first integrals.

This last result is announced in section 5 .

\section{Existence of formal psi-series}

In looking for psi-series of a system

$$
\frac{\mathrm{d} x}{\mathrm{~d} t}=X(x)
$$

one tries first for solutions of the form

$$
x(\tau)=\tau^{\alpha} \cdot A+\cdots,
$$

where $\tau^{\alpha} \cdot A=\left(\tau^{\alpha_{1}} A_{1}, \ldots, \tau^{\alpha_{m}} A_{m}\right), \tau=t-t_{p}, t_{p}$ being a singularity, $A$ is a vector of constants, and $\alpha:=\left(\alpha_{1}, \ldots, \alpha_{m}\right) \in \mathbb{Z}^{m}$ is a vector with integer components. In substituting $\tau^{\alpha}$ in equation (1), one can find $\alpha$ such that $X(x)$ can be separated as $X(x)=F(x)+R(x)$, where $F(x)$ contains the relevant higher order terms and $R(x)$ the lower order terms for $x=\tau^{\alpha} \cdot A: R(x)=\mathrm{o}(F(x))$. The following theorem tell us when psi-series appear:

Theorem 1 Consider the equation (1), where $X(x)=F(x)+R(x)$, as described above, is an analytic function. Assume

H1) There exists a vector $A=\left(A_{1}, \ldots, A_{m}\right)^{\top}$ with all components non-zero such that $F(A)=\alpha \cdot A$, for some $\alpha \in \mathbb{Z}^{m}$, where $\alpha \cdot A=\left(\alpha_{1} A_{1}, \ldots, \alpha_{m} A_{m}\right)$.

H2) $r_{i} \in\{1,2, \ldots\}$, where $\left\{-1, r_{1}, \ldots, r_{m-1}\right\}$ are different eigenvalues of $D F(A)-\operatorname{diag}(\alpha)$.

Then, there exists a (formal) m-parametric family of psi-series

$$
x(t)=\tau^{\alpha} \cdot A+\sum_{n \geq 1} \tau^{\alpha} \cdot p_{n}\left(\log \left(\frac{1}{\tau}\right)\right) \tau^{n} .
$$

\section{Sketch of the proof:}

If we differentiate expansion (2) with respect to $t$ and use the Taylor expansion of $F(x)=F\left(\tau^{\alpha} \cdot A+\cdots\right)$, we get that $\alpha \cdot A=F(A)$, which due to hypothesis H1) has at least a solution with all components non-zero.

Differentiating twice expansion (2), and taking into account again the Taylor expansion of $F(x)=F\left(\tau^{\alpha} \cdot A+\cdots\right)$, we get that $\alpha \cdot A$ is an eigenvector of eigenvalue -1 of $D F(A)-\operatorname{diag}(\alpha)$.

Now it is very convenient to perform a change of time $s=-\log \tau$, or $\tau=\mathrm{e}^{-s}$, where $\tau=t-t_{p}$, and a change of variables

$$
x=\mathrm{e}^{-s \alpha} \cdot A+\mathrm{e}^{-s \alpha} \cdot(B w),
$$

where $\mathrm{e}^{-s \alpha} \cdot v$ denotes $\left(\mathrm{e}^{-s \alpha_{1}} v_{1}, \ldots, \mathrm{e}^{-s \alpha_{m}} v_{m}\right)$, for $v=\left(v_{1}, \ldots, v_{m}\right) \in \mathbb{C}^{m}, w$ is the new variable and $B$ is the matrix formed with eigenvectors of $D F(A)-\operatorname{diag}(\alpha)$. 
Denoting ${ }^{\prime}=\mathrm{d} / \mathrm{d} s$, then $\mathrm{d} / \mathrm{d} t=-\mathrm{e}^{s}{ }^{\prime}$, and it follows that the equation for the new variables $w$ is

$$
\left.w^{\prime}+\operatorname{diag}\left(-1, r_{1}, \ldots, r_{m-1}\right) w=\text { (lower order terms }\right) .
$$

Introducing $w_{n}(s)=B^{-1} p_{n}$, the psi-series (3) takes the form

$$
x(s)=\mathrm{e}^{-s \alpha} \cdot A+\sum_{n \geq 1}\left(\mathrm{e}^{-s \alpha} \cdot p_{n}\right) \mathrm{e}^{-n s}=\mathrm{e}^{-s \alpha} \cdot A+\sum_{n \geq 1}\left(\mathrm{e}^{-s \alpha} \cdot\left(B w_{n}\right)\right) \mathrm{e}^{-n s},
$$

and hence, by change (4), we look for an expansion of $w$ of the following form

$$
w(s)=\sum_{n \geq 1} w_{n}(s) \mathrm{e}^{-n s},
$$

$w_{n}$ being polynomials in the variable $s$.

Taking into account that $w^{\prime}=\sum_{n \geq 1}\left(w_{n}^{\prime}-n w_{n}\right) \mathrm{e}^{-n s}$, we get the differential equation for $w_{n}$

$$
w_{n}^{\prime}+\Lambda_{n} w_{n}=c_{n},
$$

where $\Lambda_{n}=\operatorname{diag}\left(-1, r_{1}, \ldots, r_{m-1}\right)-n$ Id and $c_{n}$ is a polynomial function that depends only on $w_{k}, k=1, \ldots, n-1$.

The next step is to obtain the polynomial vector $w_{n}$.

If $n<\min \left\{r_{1}, \ldots, r_{m-1}\right\}$, by induction, we get that the only polynomial solution of the equation (7) is the constant vector $w_{n}=-\Lambda_{n}{ }^{-1} c_{n}$, since $\Lambda_{n}$ is an invertible matrix. So, $p_{n}=B w_{n}$ is a constant vector too.

If $n=r_{i}, i=1, \ldots, m-1$, the matrix $\Lambda_{n}$ is singular and it has eigenvalues $n+1$, $n-r_{1}, \ldots, 0^{i}, \ldots, n-r_{m-1}$. In this case, we have that the $i$-component of the differential equation $(7)$ is

$$
w_{r_{i}, i}^{\prime}=c_{r_{i}, i} .
$$

So, the solution of the above equation is constant unless $c_{r_{i}, i} \neq 0$. In the case where $c_{r_{i}, i}=0, \forall i=1, \ldots, m-1$, the polynomial solution of the equation ( 7 ) is constant for all $n$ because $\Lambda_{n}$ is a non singular matrix for $n \neq r_{i}, i=1, \ldots, m-1$. Under these circumstances, the expansion of $x(t)$ are Laurent series. According to the Painlevé property, system (1) is candidate to be an integrable system.

On the other hand, if there exists $i \in\{1, \ldots, m-1\}$ such that $c_{r_{i}, i} \neq 0$, the solutions of the equation ( 7 ) are non constants polynomials vectors and genuine psi-series appear. $\square$

\section{Convergence of the psi-series}

From now on, we shall suppose that the vector field $X(x)$ is a polynomial vector. In this case, the vector $c_{n}$ of the formula (7) can be written as:

$$
c_{n}=\sum_{j=2}^{k} \sum_{i_{1}+\cdots+i_{j}=n} R_{j}\left[B w_{i_{1}}, \ldots, B w_{i_{j}}\right],
$$

where $R_{j}$ is a $j$-linear form.

The following theorem give us a real region depending of two parameters: $C$ and $K$, where $C$ appears in the following lemma: 
Lemma 2 Let $j$ be an integer number. So, there exists a constant $C$ such that:

$$
a_{n}:=\sum_{i_{1}+\cdots+i_{j}=n} \frac{1}{\left(i_{1}+1\right) \ldots\left(i_{j}+1\right)} \leq \frac{C}{(n+1)^{\beta}},
$$

with $0<\beta<1$.

Theorem 3 Let $n_{0}$ be the integer such that the following inequality is true for $n \geq n_{0}$ :

$$
\frac{C \sum_{j=2}^{k}\left\|R_{j}\right\|\|B\|^{j}}{(n+1)^{\beta}} \cdot \frac{3}{n}<\frac{1}{n+1},
$$

where $0<\beta<1$ is the exponent that appears in lemma 2.

Let $K$ be a real positive number that satisfies the following finite number of inequalities:

$$
\left\|w_{n}\right\| \leq(n+1)^{-1}(2 K+K s)^{\frac{n}{r}}, n=0,1, \ldots, \max \left\{n_{0}, 4 r\right\},
$$

and $r=\max \left\{r_{1}, \ldots, r_{m-1}\right\}$.

Then the psi-series $\sum w_{n}(s) \mathrm{e}^{-n s}$ and $\sum p_{n}(s) \mathrm{e}^{-n s}$ are convergent for real $s>s_{0}$, where $s_{0}$ is the positive root of the equation:

$$
K(2+s)=\mathrm{e}^{r s} .
$$

Remark 4 The study of convergence for a complex value $s_{c}=s-i \theta, s \in \mathbb{R}$ and $\theta \in[0,2 \pi)$, can be reduced to the real case [4].

\section{How to find singularities using psi-series}

Our aim is to find the singularity $t_{p}$ and the others free constants $c_{1}, \ldots, c_{m-1}$ of system (1) of the solution with initial conditions $x\left(t_{0}\right)=x_{0}$.

To solve the problem, we suppose that we know $x(t)$, where $t$ belongs to the region of convergence of the psi-series expansion. We can compute $x(t)$ by using a standard numerical integration method like Runge-Kutta $7-8$.

Next, we consider the following application: (we identify $\mathbb{C} \equiv \mathbb{R}^{2}$ )

$$
\begin{aligned}
F_{\Delta t}: & \mathbb{R}^{2 m} \longrightarrow \mathbb{R}^{2 m} \\
& \left(t_{p}, c_{1}, \ldots, c_{m-1}\right) \longrightarrow x(t)=\left(\tau^{\alpha} \cdot A+\sum_{n \geq 1} \tau^{\alpha} \cdot p_{n}\left(\log \frac{1}{\tau}\right) \tau^{n}\right),
\end{aligned}
$$

where $\Delta t=t-t_{p}$ small.

So, we have to solve numerically the following numerical equation in $\mathbb{R}^{2 m}$ :

$$
F_{\Delta t}\left(t_{p}, c_{1}, \ldots, c_{m-1}\right)=(x(t)) .
$$

We can solve it by using, for example, the Newton-Raphson method. For a more details about this section, see [4].

\section{Relation between psi-series and General Integrals}

In [4] we have studied all the integrable quadratic systems with a center at the origin. We have proved by using the results of Lunkevich and Sibirskii [3] that if the General Integral 
of the system is algebraic, then the only possible psi-series expansions reduce to Laurent series.

We have here an analogous result.

Theorem 5 Let

$$
\frac{\mathrm{d} x}{\mathrm{~d} t}=X(x)
$$

be a differential system of dimension $m$ where $X(x)$ is a polynomial vector field.

Assume that a solution $x(t)$ of (11) and a singularity $t_{p}$ of $x(t)$, has a psi-series expansion as a function of $\tau=t-t_{p}$

$$
x(t)=\tau^{\alpha} \cdot A+\sum_{n \geq 1}\left(\tau^{\alpha} \cdot p_{n}\left(\log \frac{1}{\tau}\right)\right) \tau^{n} .
$$

Suppose that (11) is an integrable system and the $m-1$ general integrals are algebraic integrals:

$$
\begin{array}{cc}
Q_{1}(x, H) & =0, \\
\vdots & \vdots \\
Q_{m-1}(x, H) & =0,
\end{array}
$$

where $Q_{i}$ are polynomials in the variables $x$ and $H=\left(H_{1}, \ldots, H_{m-1}\right)^{\top}$ (free constants of integration).

Then this psi-series is in fact a Laurent series (all the polynomial $p_{n}$ are simply constants).

In few words, polynomial systems with genuine psi-series cannot have a complete set of algebraic integrals.

\section{Acknowledgements}

Research by Amadeu Delshams is partially supported by the spanish grant DGICYT PB94-0215, the EC grant ERBCHRXCT940460 and the catalan grant CIRIT GRQ931135.

\section{References}

1. L. Gavrilov. Non-integrability of the equations of heavy gyrostat. Compositio Mathematica, 82:275291, 1992.

2. E.L. Hille. Ordinary Differential Equations in the Complex Domain. Wiley-Interscience, New York, 1976.

3. V. A. Lumkevich and K. S. Sibirskii. Integrals of a general quadratic differential systems in cases of a center. Differential Equations, 18(5):563-568, 1982.

4. A. Mir and A. Delshams. Psi-series of quadratic systems on the plane. Preprint, 1995.

5. A. Ramani, B. Grammaticos, and T. Bountis. The Painlevé property and singularity analysis of integrable and non-integrable systems. Phys. Reports, 180(3):159-245, 1989. 